\title{
Targeted hypoxia reduction restores T cell infiltration and sensitizes prostate cancer to immunotherapy
}

\author{
Priyamvada Jayaprakash, ${ }^{1}$ Midan Ai, ${ }^{1}$ Arthur Liu, ${ }^{1,2}$ Pratha Budhani, ${ }^{1}$ Todd Bartkowiak, ${ }^{1,2}$ Jie Sheng, ${ }^{1}$ Casey Ager, ${ }^{1,2}$ \\ Courtney Nicholas, ${ }^{1}$ Ashvin R. Jaiswal, ${ }^{1}$ Yanqiu Sun, ${ }^{1}$ Krishna Shah, ${ }^{1}$ Sadhana Balasubramanyam, ${ }^{1}$ Nan Li, ${ }^{3}$ Guocan Wang, ${ }^{4}$ \\ Jing Ning, ${ }^{3}$ Anna Zal, ${ }^{1}$ Tomasz Zal, ${ }^{1,2}$ and Michael A. Curran ${ }^{1,2}$
}

'Department of Immunology, University of Texas MD Anderson Cancer Center, Houston, Texas, USA. ${ }^{2}$ University of Texas Health Science Center at Houston Graduate School of Biomedical Science, Houston, Texas, USA. ${ }^{3}$ Department of Biostatistics and ${ }^{4}$ Department of Cancer Biology, University of Texas MD Anderson Cancer Center, Houston, Texas, USA.

\begin{abstract}
Despite the success of immune checkpoint blockade against melanoma, many "cold" tumors like prostate cancer remain unresponsive. We found that hypoxic zones were prevalent across preclinical prostate cancer and resisted $\mathrm{T}$ cell infiltration even in the context of CTLA-4 and PD-1 blockade. We demonstrated that the hypoxia-activated prodrug TH-302 reduces or eliminates hypoxia in these tumors. Combination therapy with this hypoxia-prodrug and checkpoint blockade cooperated to cure more than $\mathbf{8 0} \%$ of tumors in the transgenic adenocarcinoma of the mouse prostate-derived (TRAMP-derived) TRAMP-C2 model. Immunofluorescence imaging showed that TH-302 drives an influx of T cells into hypoxic zones, which were expanded by checkpoint blockade. Further, combination therapy reduced myeloid-derived suppressor cell density by more than $\mathbf{5 0} \%$, and durably reduced the capacity of the tumor to replenish the granulocytic subset. Spontaneous prostate tumors in TRAMP transgenic mice, which completely resist checkpoint blockade, showed minimal adenocarcinoma tumor burden at 36 weeks of age and no evidence of neuroendocrine tumors with combination therapy. Survival of $\mathrm{Pb}$-Cre4,

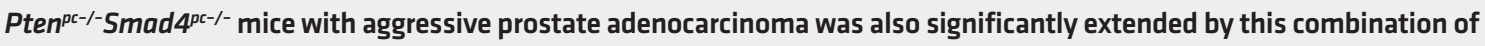
hypoxia-prodrug and checkpoint blockade. Hypoxia disruption and T cell checkpoint blockade may sensitize some of the most therapeutically resistant cancers to immunotherapy.
\end{abstract}

\section{Introduction}

We have shown previously that antibody blockade of the $\mathrm{T}$ cell immune checkpoint receptors CTLA-4 and PD-1 synergized in curing mice of preimplanted B16 melanoma (1). In clinical trials, more than $50 \%$ of metastatic melanoma patients receiving this combination therapy achieved objective clinical responses, with the majority experiencing more than $85 \%$ reduction in tumor burden (2). For melanoma patients, immunotherapy now offers the hope of durable remissions and even lifelong cures in a setting in which therapeutic success was previously defined by a few months of extended survival. For patients suffering from more therapeutically resistant solid tumors such as castration-resistant prostate cancer, pancreatic cancer, and nonhypermutated colorectal cancer, however, no such curative hope exists, as T cell checkpoint blockade shows little efficacy $(3,4)$. Our studies of murine tumor models of prostatic adenocarcinoma suggest that, unlike melanoma, these tumors effectively prevent accumulation of tumorspecific $\mathrm{T}$ cells through establishment of inaccessible hypoxic

\section{Related Commentary: p. 4761}

Authorship note: PJ and MA contributed equally to this work. Conflict of interest: The authors have declared that no conflict of interest exists. License: Copyright 2018, American Society for Clinical Investigation.

Submitted: July 13, 2017; Accepted: August 30, 2018

Reference information: J Clin Invest. 2018;128(11):5137-5149.

https://doi.org/10.1172/JCI96268. cores, recruitment of myeloid suppressor cells, and establishment of dense stromal barriers lacking in relevant tumor antigens. Tumor hypoxia, in particular, predicts poor outcome across all cancers (5), and serves as a center for recruitment, polarization, and expansion of immune-suppressive stromal cell populations (6-9). For prostate cancer, increased hypoxia has been shown to strongly correlate with more aggressive disease (10), and to be both prevalent and severe in prostate patients (11).

Antibodies that block $\mathrm{T}$ cell coinhibitory receptors or activate costimulatory ones can potently reverse $\mathrm{T}$ cell anergy and suppression in "hot" tumors with preexisting immune infiltrates; however, immunotherapy performs poorly in "cold" tumors in which T cells are sparse or absent from the tumor microenvironment. We find few, if any, T cells infiltrating the hypoxic areas of either transplantable or spontaneous syngeneic models of prostate cancer. Hypoxia acts both directly (e.g., HIF-1 $\alpha$-mediated upregulation of VEGF) and indirectly (recruitment of cells that promote abnormal angiogenesis) to promote dysregulated tumor angiogenesis. The resulting vasculature expresses too little of the adhesion molecules necessary to support T cell extravasation and can be programmed by hypoxia-induced factors to actively induce apoptosis of T cells via FAS receptor engagement (12). Those T cells that are able to enter hypoxic tumors face a metabolically hostile and highly suppressive environment characterized by acidic $\mathrm{pH}$, depletion of essential amino acids and nutrients (e.g., arginine, tryptophan, glucose), dense expression of inhibitory ligands (e.g., PD-L1), extracellular adenosine, and high concentrations of suppressive 
cytokines (e.g., TGF- $\beta$ ) (13). In addition, hypoxia drives recruitment of immature myeloid cells, and fosters their phenotypic conversion into highly suppressive myeloid-derived suppressor cells (MDSCs) and tumor-associated macrophages (6). Thus, hypoxia plays a prominent role in both the establishment and the maintenance of tumor immune privilege.

We chose to utilize the hypoxia-activated prodrug TH-302 (evofosfamide) as a mechanism for disruption of tumor hypoxia. $\mathrm{TH}-302$ is nonlymphotoxic in animal studies and clinical trials, and we find that it can be administered concurrently with immunotherapy without harming the antitumor $\mathrm{T}$ cell response (14). Critically, we observed that hypoxia was reduced and tumor vasculature normalized after TH-302 treatment. Hypoxia reduction and checkpoint blockade combined to cure $80 \%$ of animals implanted with TRAMP-C2 prostate cancer. We found that this hypoxia-targeted therapy promoted a dramatic and durable decline in MDSCs in the tumor, which acted in concert with checkpoint blockade to promote elevated $\mathrm{CD}^{+} / \mathrm{MDSC}$ ratios and tumor elimination. In mice receiving $\mathrm{TH}-302$ with $\mathrm{T}$ cell checkpoint blockade, $\mathrm{CD}^{+} \mathrm{T}$ cell proliferation (Ki67), cytotoxic potential (granzyme B), activation (CD44), and effector cytokine production (IFN- $\gamma$ and TNF- $\alpha$ ) all increased. At the same time proliferation of granulocytic MDSCs in hypoxic zones of prostate tumors decreased, as did their capacity to suppress $\mathrm{T}$ cell proliferation.

Transgenic adenocarcinoma of the mouse prostate (TRAMP) transgenic mice develop tumors in the dorsolateral prostate, which metastasize to the periaortic nodes and lungs, with occasional metastases to the kidney, adrenal gland, and bone as early as 12 weeks (15). Castration resistance develops at varied rates, but nearly $100 \%$ of these animals are fully androgen-independent by 20 weeks (16). On the C57BL/6 background, approximately $80 \%$ of these animals develop adenocarcinomas, while $20 \%$ develop neuroendocrine carcinomas (17). Much like metastatic prostate cancer patients, these mice are entirely resistant to checkpoint blockade. We found, however, that hypoxia ablation combined with checkpoint blockade produced profound therapeutic responses in these animals, with most exhibiting low to unmeasurable tumor burden even 3 months after discontinuation of therapy. These findings further illustrate that disruption of hypoxic zones compromises the integrity of the suppressive stromal myeloid network and can sensitize even the most therapeutically resistant preclinical model of prostate cancer to $\mathrm{T}$ cell checkpoint immunotherapy.

\section{Results}

Hypoxic zones within prostate tumors lack T cells. Hypoxia is known to be a common feature of clinical prostate cancer that correlates with poor prognosis (10), and we have found it to be equally pervasive across murine transplantable and spontaneous preclinical models (Supplemental Figure 1A; supplemental material available online with this article; https://doi.org/10.1172/JCI96268DS1). In the TRAMP-C2 model, mice bearing $200-$ to $300-\mathrm{mm}^{3}$ prostate tumors were sacrificed following administration of the hypoxia-marking compound pimonidazole and the distribution of T cells within them examined by immunofluorescence imaging. Normoxic zones of these tumors were infiltrated by both $\mathrm{CD}^{+}$and $\mathrm{CD}^{+}{ }^{+} \mathrm{T}$ cells, although with a sufficient fraction of $\mathrm{CD} 4^{+} \mathrm{FoxP} 3^{+}$ Tregs to functionally suppress the effector populations (Supple- mental Figure 1B). In contrast, the hypoxic zones of this same tumor lacked substantial infiltration by any type of $\mathrm{T}$ cells. Within the more physiologic setting of spontaneous prostate cancer in TRAMP transgenic mice, we found that, just as in TRAMP-C2, $\mathrm{T}$ cells were largely excluded from hypoxic zones (Supplemental Figure 1C). This lack of $\mathrm{T}$ cell infiltration of hypoxic zones led us to hypothesize that they may serve as islands of immune privilege and, potentially, immunotherapy resistance within the tumor microenvironment of these cancers.

Targeted hypoxia reduction cooperates with $T$ cell checkpoint blockade to promote rejection of TRAMP-C2 prostate tumors. To test the hypothesis that reduction of hypoxia might reduce prostate cancer immune resistance, we used the hypoxia-activated prodrug compound $\mathrm{TH}-302$ as a nonlymphotoxic means to reduce hypoxia. TH-302 is cytotoxic within hypoxic zones of tumors; however, as we found few or no $\mathrm{T}$ cells in these areas, we did not anticipate bystander $\mathrm{T}$ cell killing. In addition, $\mathrm{TH}-302$ has an exceptionally short in vivo half-life and selectively kills proliferating cells, whereas we found little proliferation among the few $\mathrm{T}$ cells persisting under hypoxia. Mice were implanted with subcutaneous TRAMP-C2 tumors and treated with either combination antibody blockade of the CTLA-4 and PD-1 immune checkpoint receptors, $\mathrm{TH}-302$ hypoxia-activated prodrug, or the prodrug/checkpoint combination in 2 cycles starting 7 days later (Supplemental Figure 2A). The combination of TH-302 with $\alpha$ CTLA $-4 / \alpha \mathrm{PD}-1$, which confers $82 \%$ overall survival (OS), demonstrated therapeutic superiority to $\mathrm{TH}-302$ alone $(P<0.001$; OS 30\%) and to dual antibody alone ( $P=0.016$; OS 55\%) (Figure 1A). The same hierarchy of efficacy was reflected in measurements of tumor growth $(P<0.0001$ for combination versus each monotherapy). We chose to focus on the combination of TH-302 $+\alpha \mathrm{CT}$ LA $-4 / \alpha \mathrm{PD}-1$ because of its high potential for clinical translation and greater efficacy than TH-302 $+\alpha$ CTLA -4 (OS 50\%) or $+\alpha$ PD-1 (OS 30\%) alone (Supplemental Figure 2B). The capacity of TH-302 to cure some animals in this study was surprising given that its cytotoxic effects are confined to areas of tumor hypoxia. To determine whether disruption of these hypoxic zones was catalyzing an immune response capable of eliminating the entirety of the tumor, we repeated these experiments in immune-deficient Rag-knockout mice and found that neither TH-302 nor the combination could cure any animals (Supplemental Figure 2C). The combination of TH-302 and $\alpha$ CTLA- $4 / \alpha$ PD- 1 also demonstrated a significant therapeutic benefit in terms of survival $(P=0.02)$ and tumor growth control $(P<0.0001)$ versus untreated animals against MyC-CaP prostate tumors preimplanted 21 days earlier (Supplemental Figure 2D and refs. 18,19). Neither drug nor antibody monotherapy has significant benefit in this model.

While the cytotoxic nature of $\mathrm{TH}-302$ had the potential to boost $\mathrm{T}$ cell immunity through tumor and stromal depletion and antigen release from within hypoxic areas, these effects were short-lived, and the therapeutic benefit with immunotherapy could not be replicated by substitution of the untargeted parental chemotherapy drug ifosfamide (Figure 1B). Failure of ifosfamide in this context was not due to lymphotoxicity, as $\mathrm{CD}^{+} \mathrm{T}$ cells from OT-I T cell transgenic mice (ovalbumin specific) treated for 1 week with ifosfamide $(50 \mathrm{mg} / \mathrm{kg}$ ) showed equivalent capacity to expand in response to ex vivo peptide stimulation (ovalbumin-derived SIINFEKL peptide) compared with those from untreated animals 
A

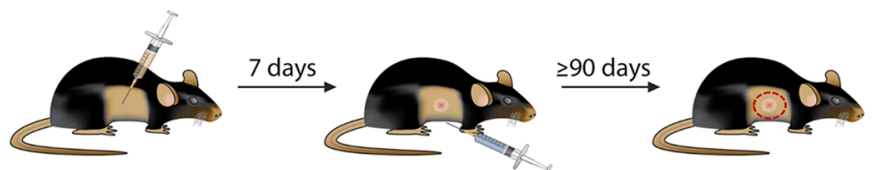

$1 \times 10^{6}$ TRAMP-C2

TH-302: d7-11,19-23

Measure w/ calipers

Ab: d7, 10, 13, 19, 22, 25
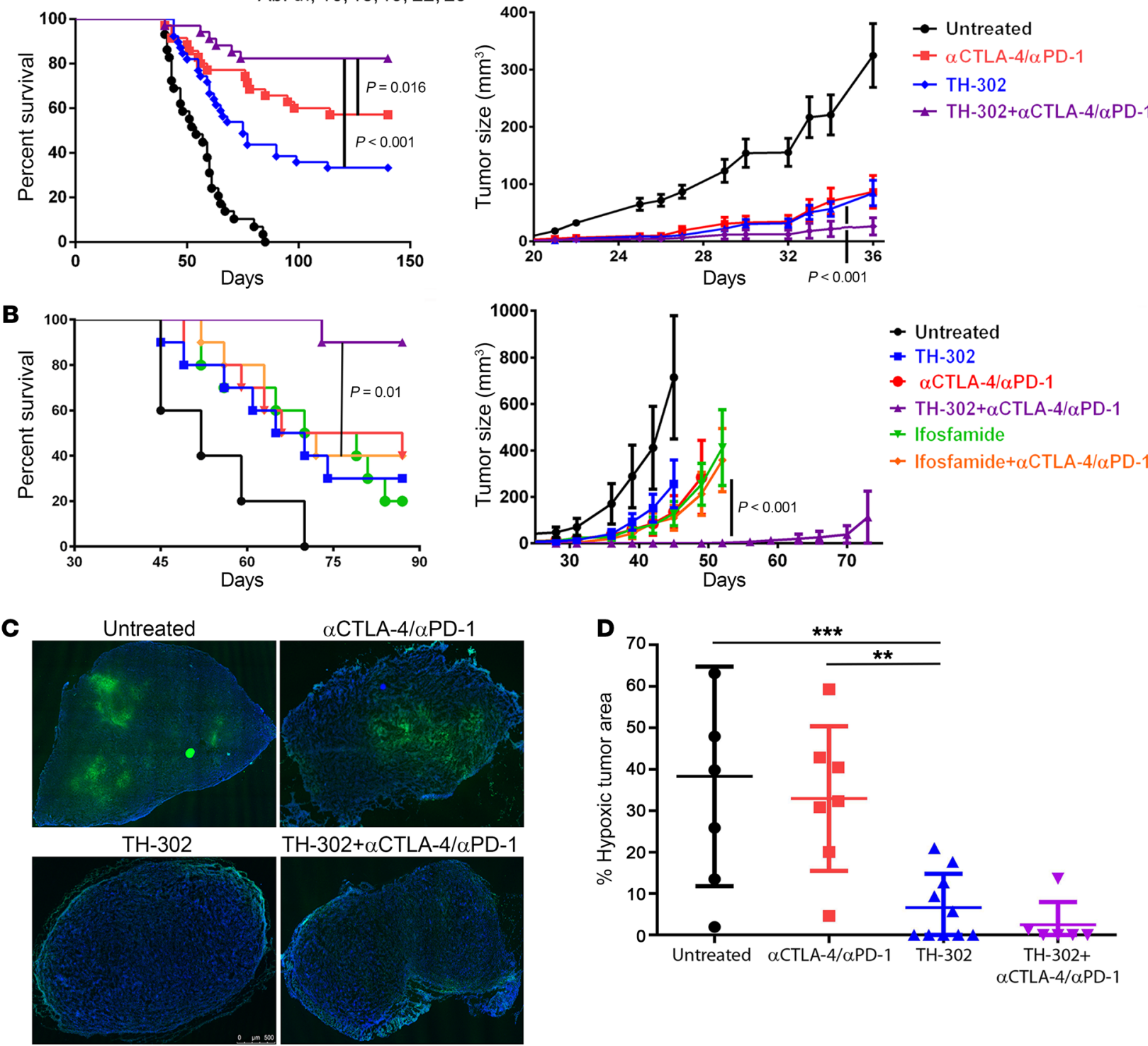

- Untreated

- TH-302

- $\alpha C T L A-4 / \alpha P D-1$

- TH-302+ $\alpha$ CTLA-4/ $\alpha$ PD- 1

* Ifosfamide

$\rightarrow$ Ifosfamide $+\alpha$ CTLA-4/ $\alpha$ PD-1

Figure 1. Hypoxia ablation cooperates with T cell checkpoint blockade to promote rejection of TRAMP-C2 prostate tumors. (A) Mice bearing TRAMP-C2 tumors preimplanted 7 days earlier were treated with 2 cycles of TH-302 and/or $\alpha$ CTLA-4/ $\alpha$ PD-1 antibody and monitored for survival and tumor growth for 140 days (5-10 mice per group, $n=5$ ). Statistical significance for survival was calculated using the log-rank (Mantel-Cox) test, and for tumor growth a linear mixed model was used to analyze the longitudinal tumor size data with consideration of within-mouse correlations. (B) Mice bearing TRAMP-C2 tumors preimplanted 7 days earlier were treated as in $\mathbf{A}$ except that groups receiving ifosfamide $(50 \mathrm{mg} / \mathrm{kg}$ ) with or without antibody were included (10 mice per group [5 untreated mice], $n=1$ ). (C) TRAMP-C2 tumor-bearing mice were treated with a single cycle of therapy, and 2 days later their tumors were stained for hypoxia following pimonidazole injection (Hypoxyprobe) and imaged at low magnification ( $\times 10)$. Representative images are shown for each group. (D) Hypoxic area from 4 full tumor slices for each group was quantified using ImageJ and the statistical significance between groups determined by ANOVA. ${ }^{* *} P<0.01,{ }^{* *} P<0.001$.

(Supplemental Figure 2E). Examination of the full area of slices through TRAMP-C2 tumors following a single cycle of TH-302 therapy revealed prominent hypoxic geography across untreated as well as checkpoint-unresponsive tumors that was profoundly diminished in mice receiving the drug (Figure 1C). Across 4 full tumors under each condition, we found that more than a third of the area of untreated or antibody-treated tumors was hypoxic (Figure 1D). In contrast, mice that received $\mathrm{TH}-302$, alone or in combination with checkpoint blockade, averaged less than $7 \%$ hypoxic area across their tumors. We hypothesize that it is the removal of these zones of hypoxia that restores $\mathrm{T}$ cell infiltration, thereby sensitizing these prostate tumors to checkpoint blockade. 
A
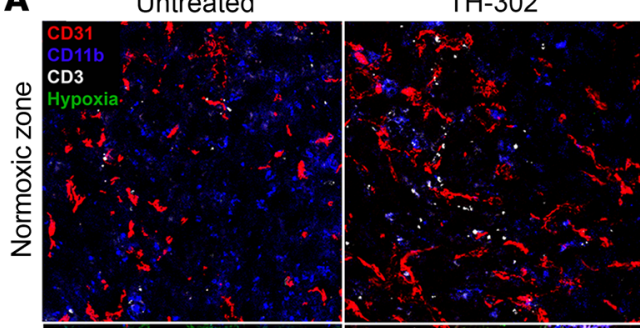

TH-302

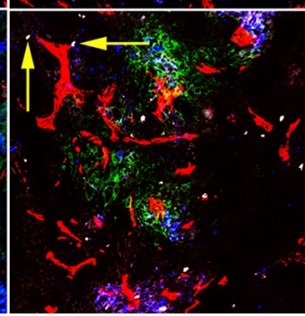

B
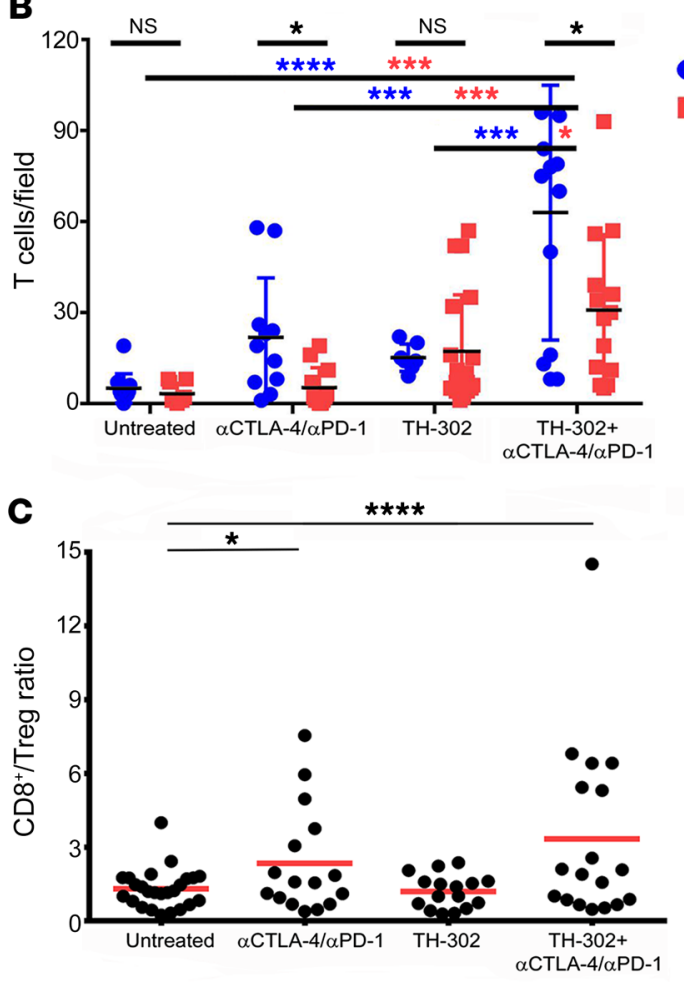

$\alpha C T L A-4 / \alpha P D-1$
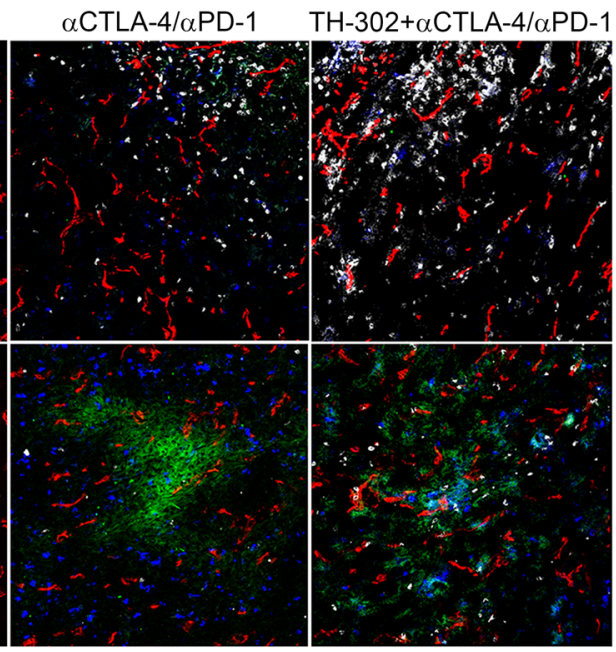

Normoxia

Hypoxia

Figure 2. Hypoxia loss reverses T cell exclusion and suppression in prostate tumors. (A) Mice bearing TRAMP-C2 tumors preimplanted 14 days earlier were treated with 1 cycle of $\mathrm{TH}-302$ and/ or antibody therapy. Tumors were isolated, OCT-mounted, frozen, sectioned, fixed, and stained for pimonidazole (FITC), CD31 (Alexa Fluor 647), CD11b (Alexa Fluor 546), and CD3 (V450). (B) Quantification of $C D 3^{+} T$ cells from tumors of mice treated in $\mathbf{A} . \mathrm{CD}^{+} \mathrm{T}$ cells in at least 10 fields per tumor were counted, and at least 2 tumors per condition were analyzed. The average $\mathrm{CD}^{+} \mathrm{T}$ cell number per field is shown. (C and D) Mice were implanted with TRAMP-C2 tumors in 30\% Matrigel and treated beginning on day 14 for 2 cycles of therapy. One day after therapy, tumor-infiltrating lymphocytes were purified and analyzed by flow cytometry. The intratumoral ratios of $\mathrm{CD}^{+} \mathrm{CD} 8$ $\mathrm{T}$ cells versus $\mathrm{CD}^{+} \mathrm{CD}^{+}{ }^{+} \mathrm{FoxP3}^{+}$Tregs (C) and CD11 b+Cr-1+arginase ${ }^{+}$MDSCs (D) are shown. $\mathbf{C}$ and $\mathbf{D}$ show individual mice from 5 independent experiments. Statistical significance between groups was determined by ANOVA. ${ }^{*} P<0.05$, ${ }^{* * *} P<0.001,{ }^{* * *} P<0.0001$.

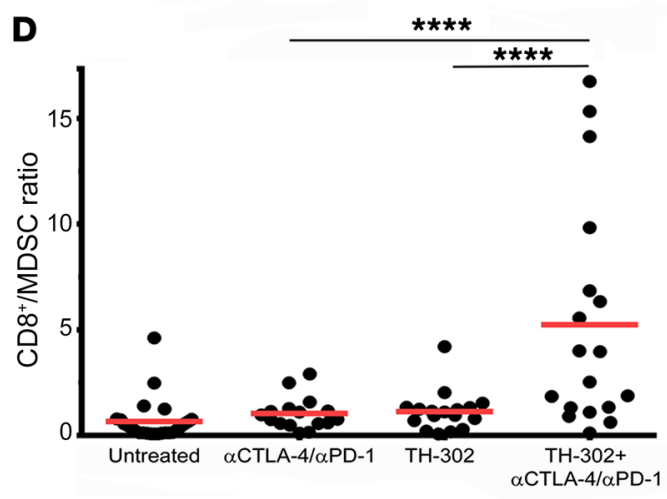

Hypoxia reduction restores $T$ cell access and increases $T$ cell proliferation within the tumor microenvironment. TRAMP-C2 tumors were established for 14 days, treated with a single cycle of therapy, and then examined for $\mathrm{T}$ cell and myeloid cell infiltration of normoxic versus hypoxic areas. As observed previously, $\mathrm{T}$ cells were present at low abundance in normoxic tumor areas but absent from hypoxic zones in untreated tumors (Figure 2A). As hypoxic zones break down following $\mathrm{TH}-302$ treatment, the capacity of $\mathrm{T}$ cells to extravasate out from vessels in these areas into the tumor tissue may improve (Figure 2A, yellow arrows); however, the numbers and depth of infiltrating $\mathrm{T}$ cells remained limited, likely as a result of multiple immune-suppressive mechanisms including engagement of $\mathrm{T}$ cell coinhibitory pathways. Immune checkpoint blockade drove robust $\mathrm{T}$ cell infiltration of normoxic areas of tumor, but very few of these cells could access hypoxic zones of these cancers, further supporting their role as centers of therapy resistance. The combination of hypoxia reduction by TH-302 and checkpoint blockade restored the capacity of these T cells not only to infiltrate hypoxic areas but to persist within them and promote tumor elimination (Figure 2A, bottom right). These differences were quantified across 10 fields per condition, showing that infiltration of hypoxic areas was impaired in the context of checkpoint blockade but not TH-302 monotherapy (Figure 2B). Combination therapy demonstrated significantly elevated normoxic infiltration compared with TH-302 monotherapy and elevated hypoxic infiltration relative to checkpoint blockade. Reduction of hypoxia and restoration of $\mathrm{T}$ cell infiltration with the $\mathrm{TH}-302$ and checkpoint blockade combination correlated with increases in the density of 
A
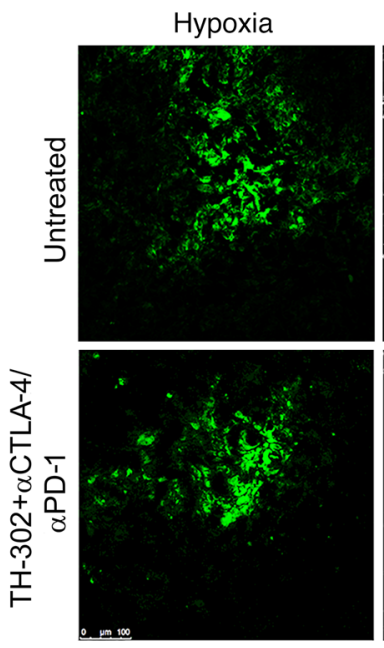

Gr-1
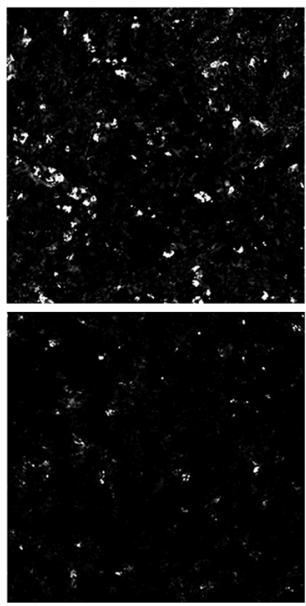

CD11b
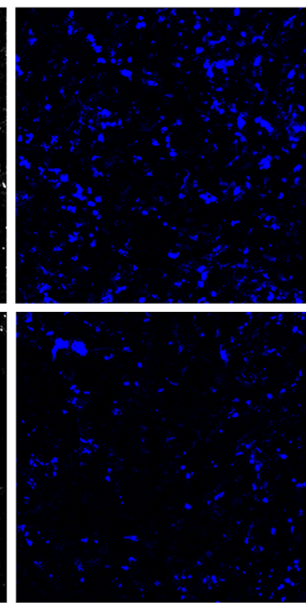

F4/80
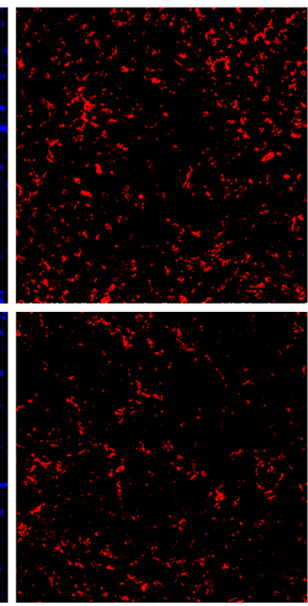

Overlay
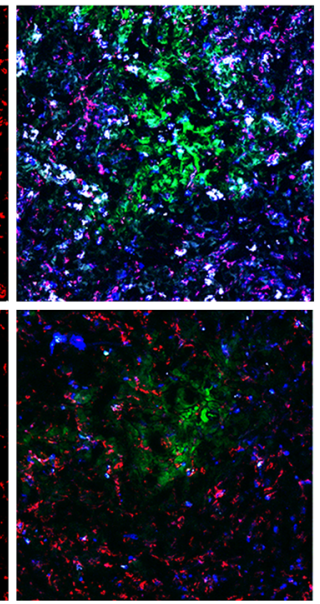

B

B
**

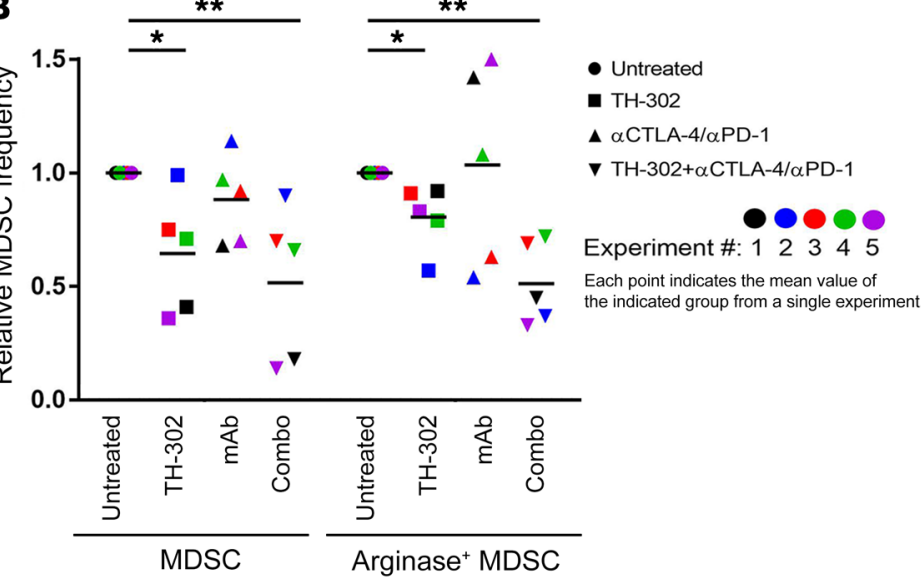

C

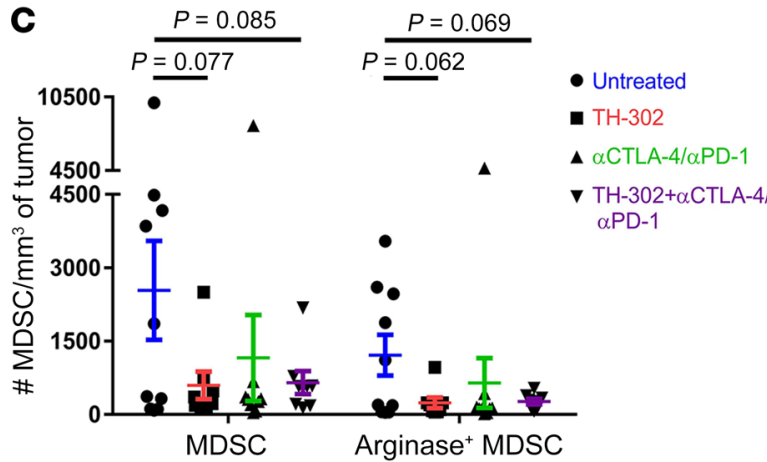

Figure 3. Hypoxia ablation reduces the frequency and density of MDSCs in the tumor microenvironment. (A) TRAMP-C2 tumors preestablished 2 weeks earlier were treated with TH-302 and antibody for 1 cycle of therapy, and then tumors were isolated, fixed, embedded in OCT, sectioned, and stained for pimonidazole (FITC), Gr-1 (V450), CD11b (Alexa Fluor 546), and F4/80 (Alexa Fluor 647). (B) Mice were implanted with TRAMP-C2 tumors in 30\% Matrigel and treated beginning on day 14 for 2 cycles of therapy. The day after conclusion of therapy, tumor-infiltrating MDSCs were purified and analyzed by flow cytometry. The frequencies of CD11 $\mathrm{b}^{+} \mathrm{Gr}-1^{+}$and $\mathrm{CD} 11 \mathrm{~b}^{+} \mathrm{Gr}-1^{+}$arginase ${ }^{+} \mathrm{MDSC}$ relative to untreated animals are shown for 5 independent experiments each with 3-10 mice per group. (C) The absolute densities of CD11b+Gr-1+ and CD11b+Gr-1+arginase+ MDSCs relative to untreated animals are shown for 2 independent experiments each with 3-10 mice per group. Statistical significance between groups was determined by ANOVA. ${ }^{*} P<0.05,{ }^{*} P<0.01$.

CD $31^{+}$vessels within these zones (Supplemental Figure 3, A and B). These healthier, more complete vessels are likely responsible for both reoxygenation of the tissue and reduction of hypoxia, as well as potentially for improved $\mathrm{T}$ cell extravasation capacity.

In order to assess the impact of hypoxia reduction on the tumor microenvironment as a whole, we established TRAMP-C2 tumors for 2 weeks, treated them with 2 cycles of therapy, and then dissociated their tumors and examined the immune infiltrates by flow cytometry. Even waiting 14 days to begin treatment, combination therapy eliminated tumor in more than $60 \%$ of animals, making isolation of sufficient numbers of tumors for analysis challenging (Supplemental Figure 4). The flow cytometry gating strategy used in analysis of these tumors, as well as relevant control gating, is shown in Supplemental Figure 5. While the ratios of $\mathrm{CD}^{+} \mathrm{T}$ cells to suppressive Tregs improved with immunotherapy in these tumors, the magnitude of that increase was small in comparison with more immunogenic cancers like melanoma (Figure 2C and refs. 1, 20,
21). Importantly, the addition of $\mathrm{TH}-302$ to checkpoint blockade did nothing to impair the benefit of checkpoint in expanding CD8 ${ }^{+}$ $\mathrm{T}$ cells relative to Tregs and myeloid-derived suppressor cells (MDSCs) (Figure 2C). Within untreated TRAMP-C2 tumors, there was approximately $1 \mathrm{Treg}$ for every $2 \mathrm{CD} 8^{+}$effector T cells; however, there were also approximately 2 arginase-positive MDSCs for every $\mathrm{CD}^{+}$cell - a potentially far greater impediment to tumor immunity (Figure 2D). Checkpoint blockade or TH-302 only minimally impacted this suppressive state, while the combination demonstrated significant synergy by increasing the $\mathrm{CD} 8^{+} / \mathrm{MDSC}$ ratio to 5:1 (Figure 2C). We hypothesize that it is the release from MDSC-mediated suppression that allows $\mathrm{CD}^{+} \mathrm{T}$ cells to expand more freely in these tumors.

Hypoxia reduction diminishes suppressive myeloid density in the tumor microenvironment. Unlike T cells, we found CD $11 \mathrm{~b}^{+} \mathrm{Gr}-1^{+}$ MDSCs, as well as CD11 b $\mathrm{Gr}-1^{-} \mathrm{F} 4 / 80^{+}$tumor-associated macrophages (TAMs), to reside in high densities within hypoxic zones of 
A

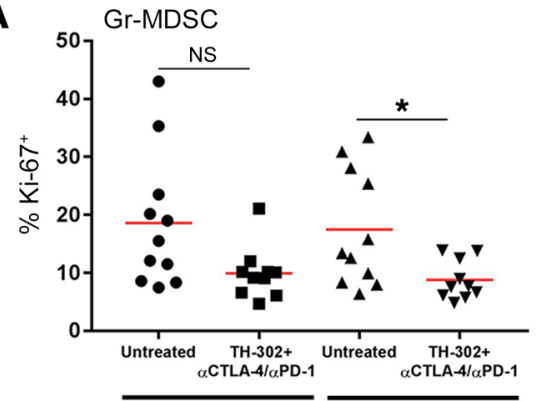

C
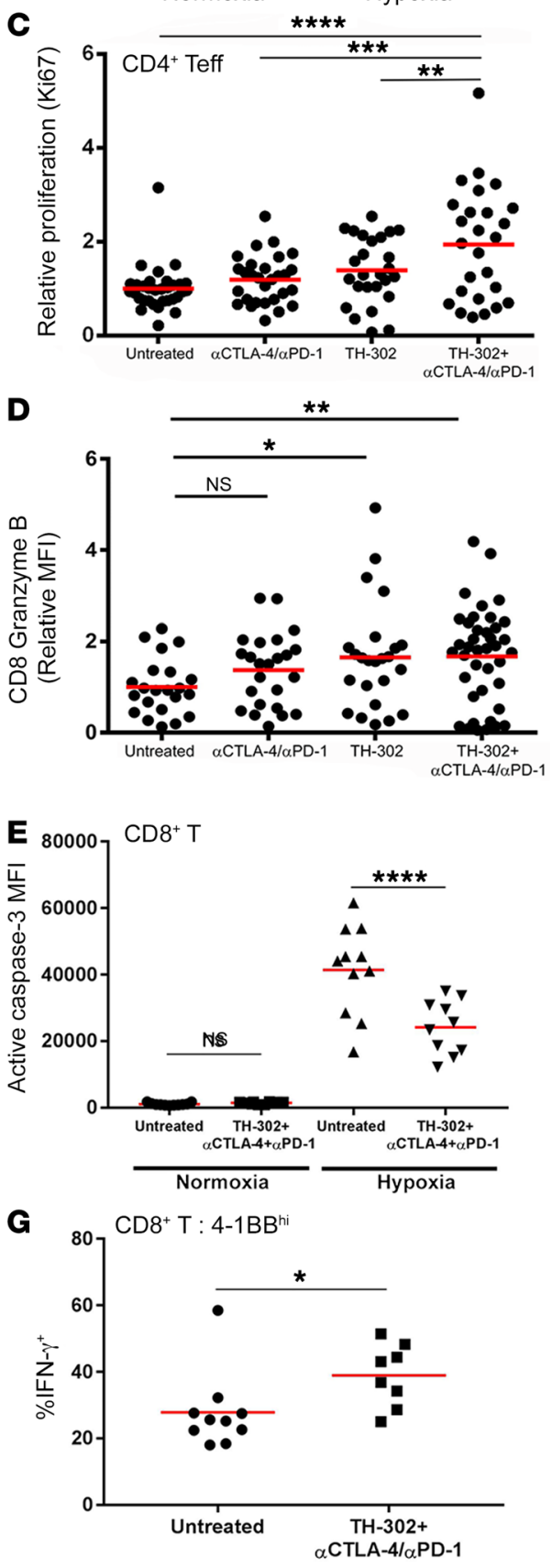
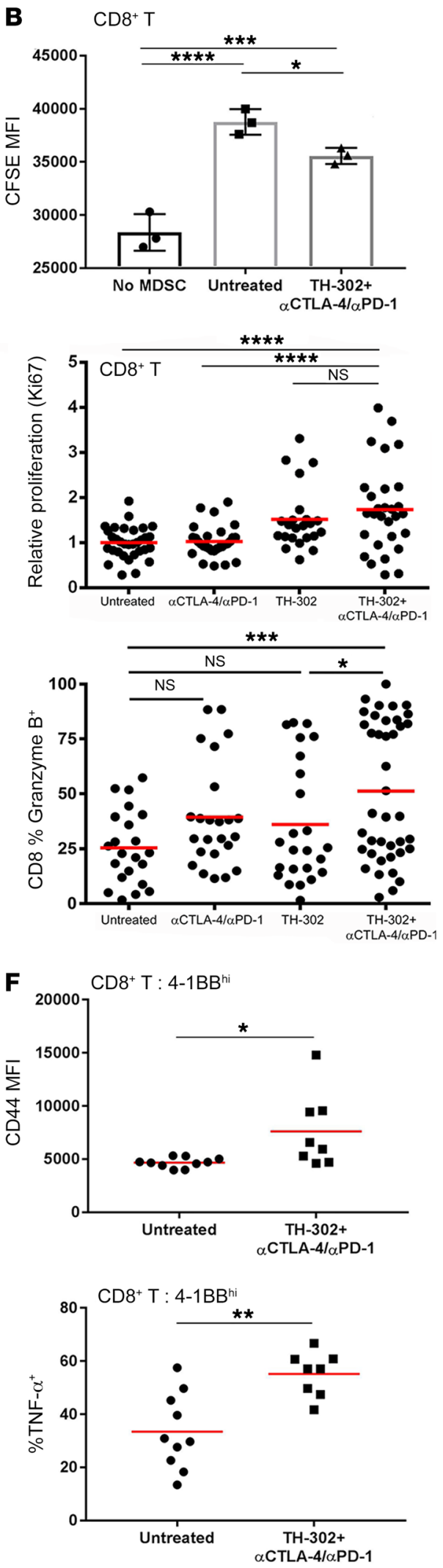

Figure 4. Combined hypoxia ablation and checkpoint blockade decreases MDSC suppressive capacity and promotes $\mathrm{T}$ cell effector function. (A) Mice were implanted with TRAMP-C2 tumors in 30\% Matrigel and treated beginning on day 21 for 1 cycle. Two days later, mice were injected with pimonidazole and euthanized 6 hours later. Proliferation of tumor-infiltrating Gr-MDSCs (CD11b+Ly6C ${ }^{+}$Ly6C $^{-}$) in pimonidazole-negative (normoxia) and pimonidazole-positive (hypoxia) regions was assessed by Ki67 staining (6-7 mice per group, $n=1$ ). (B) Mice were treated as in $\mathbf{A}$ but for $\mathbf{2}$ cycles of therapy. Two days later, tumor-infiltrating CD11 $\mathrm{b}^{+} \mathrm{Gr}-1^{+}$MDSCs were sorted and cocultured with $\alpha \mathrm{CD} 3 / \alpha \mathrm{CD} 28$-activated $T$ cells for 72 hours. T cell proliferation was assessed by CFSE dilution. Tumors from 14-16 mice with bilateral tumors were pooled for each condition. (C and $\mathbf{D})$ Mice were treated as in B but starting at day 14. The next day, tumor-infiltrating lymphocytes were purified and analyzed by flow cytometry for Ki67 (proliferation) expression (C) and granzyme B (cytotoxic potential) expression (D) $(n=5)$. Teff, effector T. (E) Mice were treated as in A, and apoptosis of tumor-infiltrating TCR $\beta^{+} \mathrm{CD} 8^{+}$ $T$ cells in pimonidazole-negative (normoxia) and pimonidazole-positive (hypoxia) regions was assessed by active caspase-3 staining (6-7 mice per group, $n=1$ ). (F and $\mathbf{G}$ ) Mice were treated as in $\mathbf{A}$. The next day, tumor-infiltrating TCR $\beta^{+} \mathrm{CD} 8^{+} T$ cells were isolated and activated for 5 hours using Leukocyte Activation Cocktail with Golgi Plug and assessed for expression of CD44 (F) and IFN- $\gamma$ and TNF- $\alpha$ (C) in the 4-1BB ${ }^{\text {med/hi }}$ subset of CD8 ${ }^{+} T$ cells (10 mice per group, $n=1$ ). Statistical significance between groups was determined by Student's $t$ test for $\mathbf{A}$ and $\mathbf{E}-\mathbf{G}$, and by ANOVA for $\mathbf{B}-\mathbf{D}$. ${ }^{*} P<0.05$, ${ }^{* *} P<0.01$, ${ }^{* *} P<0.001$, ${ }^{* * *} P<$ 0.0001 . MFI, mean fluorescence intensity.
TRAMP-C2 tumors (Figure 3A and Supplemental Figure 6A). As hypoxic zones diminished following TH-302, density of MDSCs within these areas was noticeably diminished. Following combination therapy, this loss of $\mathrm{CD}_{1} 1 \mathrm{~b}^{+} \mathrm{Gr}-1^{+}$cells was even more pronounced. In comparison with MDSC density, macrophage density within these areas remained relatively consistent (Figure $3 \mathrm{~A}$ and Supplemental Figure 6A). The tendency of MDSCs and suppressive, M2-polarized TAMs to concentrate in hypoxic zones 
A
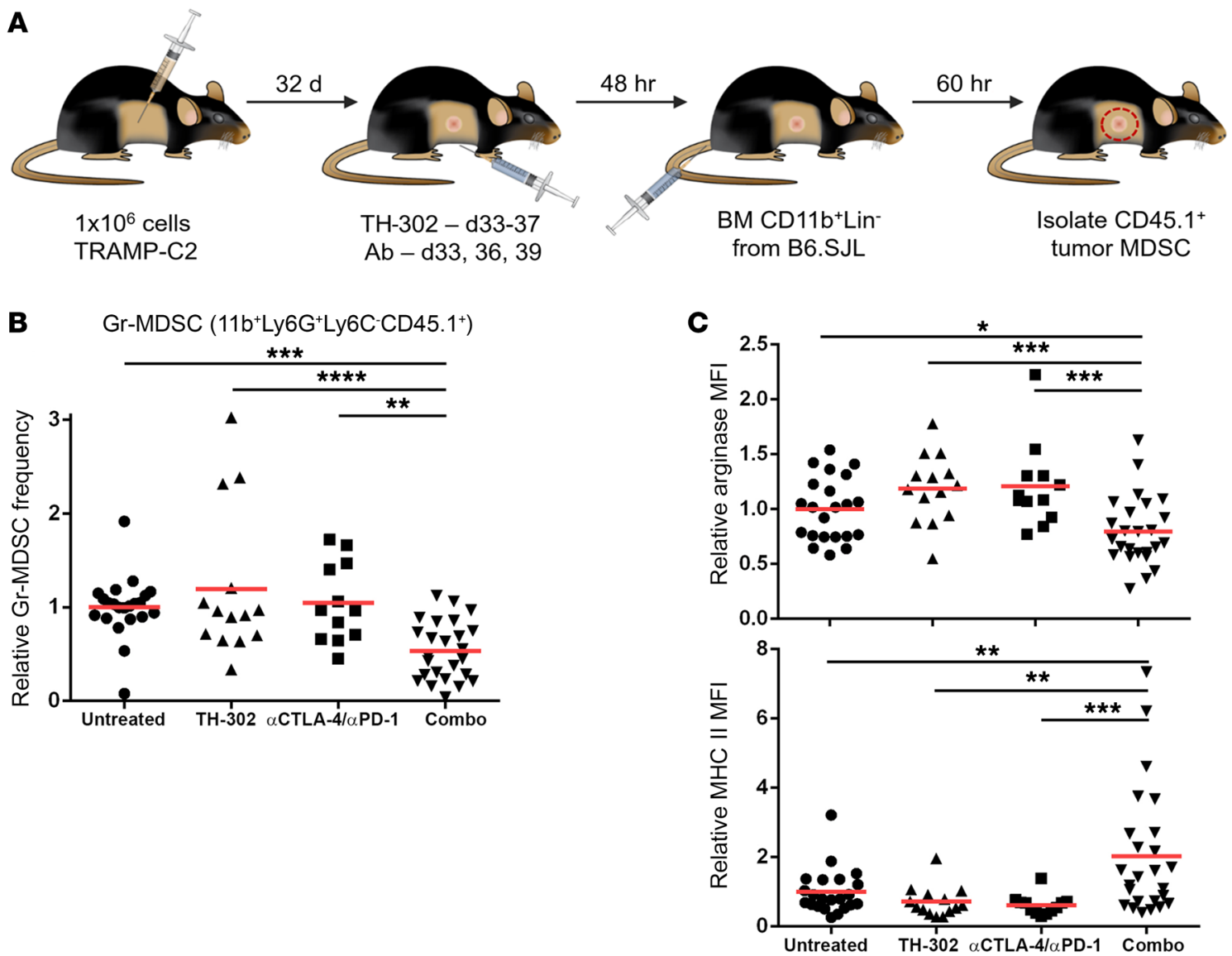

Figure 5. Hypoxia ablation and checkpoint blockade causes persistent defects in the capacity of prostate tumors to replenish their Gr-MDSCs. (A) Large, established TRAMP-C2 tumors were treated with a single cycle of therapy, rested for 2 days, and then congenically marked bone marrow myeloid progenitor cells were adoptively transferred in order to assess the influence of prior therapy on their subsequent polarization within the tumor microenvironment. (B) TRAMP-C2 tumors preestablished 32 days earlier were treated with TH-302 and antibody or left untreated, and then immature bone marrow myeloid cells (CD11 b+Lin') from B6.SJL (CD45.1+) mice were injected via tail vein. After 60 hours, tumor-infiltrating myeloid cells were isolated and analyzed by flow cytometry. The frequency of tumor-infiltrating CD45.1+ myeloid cells polarized to Gr-MDSCs (CD11 b+ Ly6C+Ly6C-CD45.1+) is shown relative to untreated animals. (C) The phenotype of Gr-MDSCs (CD11b+Ly6C + Ly6C-CD45.1+) is shown for arginase and MHC II expression (MFI relative to untreated) (5-10 mice per group, $n=2$ ). Statistical significance between groups was determined by ANOVA. ${ }^{*} P<0.05,{ }^{* *} P<0.01,{ }^{* * *} P<0.001,{ }^{* * *} P<0.0001$.

of tumors has been described; therefore, we sought to determine the global impact of loss of MDSCs from hypoxia on overall tumor MDSC density $(6,22)$.

TRAMP-C2 tumors established 2 weeks earlier were treated with 2 cycles of therapy, and then their immune infiltrates were analyzed by flow cytometry. Across 5 independent experiments, hypoxia-activated prodrug therapy alone reduced the frequency of $\mathrm{CD} 11 \mathrm{~b}^{+} \mathrm{Gr}-1^{+} \mathrm{MDSC}$ to 0.64 relative to untreated tumors, with the addition of immunotherapy further reducing this frequency to 0.51 (Figure 3B). While MDSCs have multiple mechanisms of $\mathrm{T}$ cell suppression, production of the enzyme arginase I is arguably the most potent, as $\mathrm{T}$ cells are exceptionally sensitive to concentrations of extracellular arginine. The frequency of arginase-expressing MDSCs declined to 0.83 of its baseline with $\mathrm{TH}-302$ alone and was further reduced to 0.51 with combination therapy (Figure 3B). Pooling and analyzing the individual mice rather than the experimental means from these 5 experiments also showed significant MDSC and arginase ${ }^{+}$MDSC reduction with $\mathrm{TH}-302$ or the TH-302 and checkpoint blockade combination (Supple- mental Figure 6B). In some experiments, the level of arginase ${ }^{+}$ MDSCs appeared elevated relative to baseline in mice receiving checkpoint blockade alone, suggesting a potential mechanism of adaptive immune resistance. Absolute densities of $\mathrm{CD} 11 \mathrm{~b}^{+} \mathrm{Gr}-1^{+}$ MDSCs or arginase $\mathrm{e}^{+}$MDSs were reduced to approximately $25 \%$ of their density in untreated tumors with hypoxia-activated prodrug therapy, and, in this case, no further reduction was achieved by the addition of checkpoint blockade (Figure 3C).

Combined hypoxia reduction and checkpoint blockade decreases MDSC suppressive capacity and promotes $T$ cell effector function. We next sought to dissect the impact of TH-302 and checkpoint blockade therapy on the functional properties of $\mathrm{T}$ cells, MDSCs, and tumor cells within TRAMP-C2 prostate tumors. TRAMP-C2 tumors established 21 days earlier were treated with a single cycle of TH-302 and checkpoint blockade, and then their tumor-infiltrating T cells and myeloid cells were analyzed by flow cytometry. Granulocytic MDSC (Gr-MDSC) proliferation (Ki67) decreased significantly in hypoxic zones of combination-treated versus untreated TRAMP-C2 tumors (Figure $4 \mathrm{~A}$ ). This finding is 
A

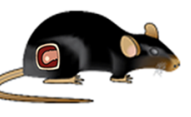

TRAMP GEMM

16 weeks old

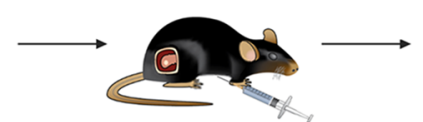

TH-302: d1-5, 12-16, 24-28, Ab: d1 $4,7,12,15,18,24,27,30$

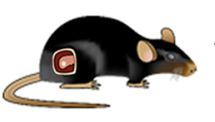

Follow survival
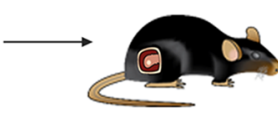

Prostate TIL analysis

36 weeks old
B

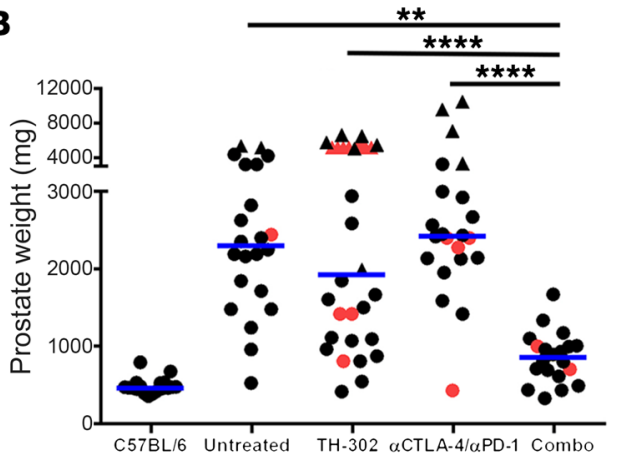

D

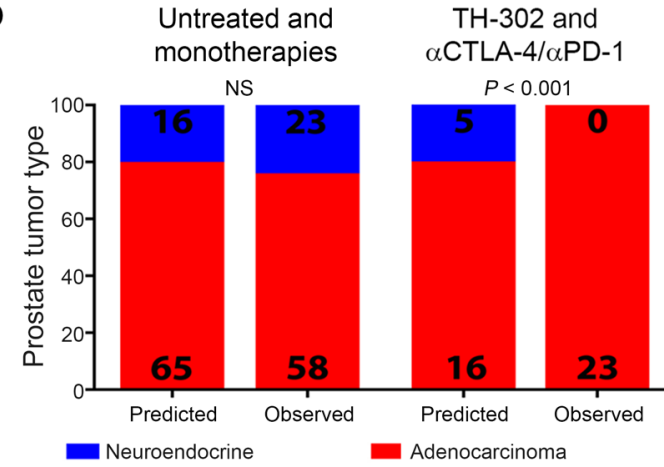

$\mathbf{F}$

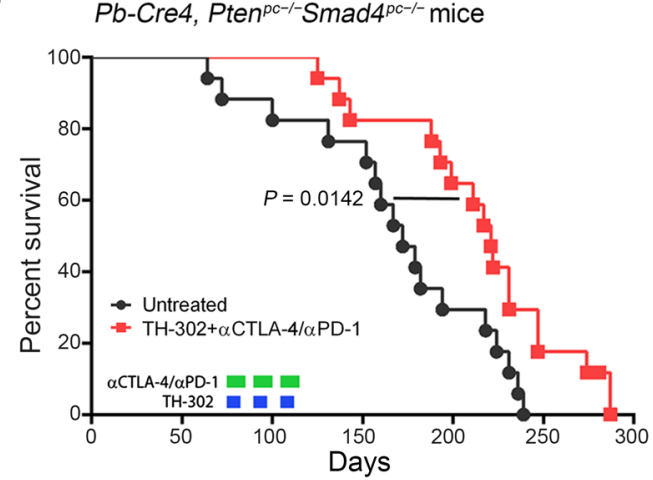

C

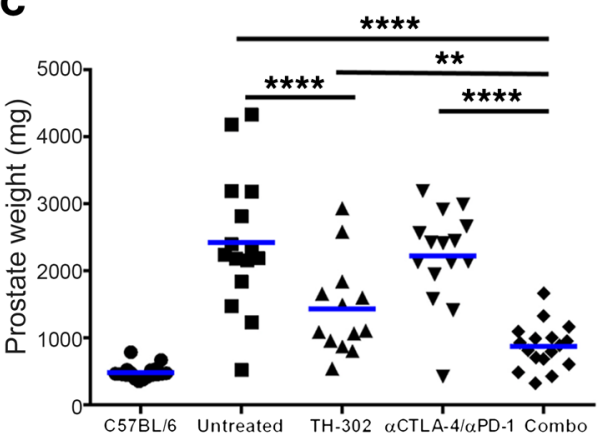

E
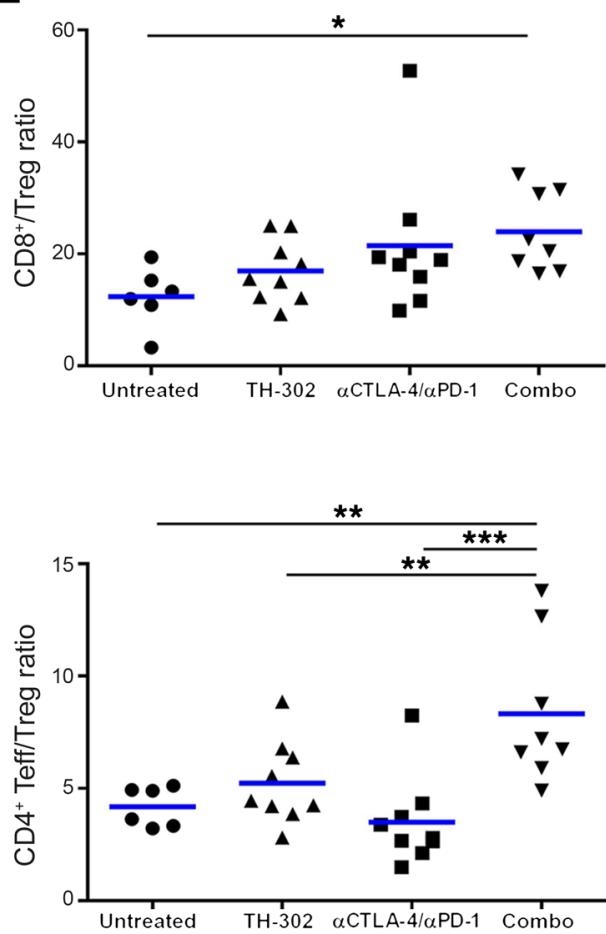

Figure 6. Hypoxia ablation and checkpoint blockade combine to control spontaneous prostate tumors in TRAMP transgenic mice. (A) TRAMP mice were treated at 16 weeks for 3 cycles with TH-302 and/or antibody and euthanized at 36 weeks and their prostates weighed. (B) Mice that died before 36 weeks are shown in red, survivors in black. Adenocarcinomas are indicated by circles and neuroendocrine carcinomas by triangles. Average weight of early-death neuroendocrine tumors (some red triangles) is shown where tumors could not be freshly weighed. (C) Only adenocarcinomas from mice that lived to 36 weeks are shown. (D) Predicted distribution of adenocarcinomas versus neuroendocrine cancer in these mice based on historical data is shown compared with the observed values for untreated + monotherapy (sum of no treatment, $\alpha$ CTLA-4/ $\alpha$ PD-1 alone, TH-302 alone) versus TH-302 $+\alpha C T L A-4 / \alpha P D-1$. $P$ values were calculated using 1-sample proportion test compared with historical 80\%:20\% adenocarcinoma/neuroendocrine frequency. (E) Prostate tissue isolated from 36-week-old TRAMP transgenic mice was dispersed into single-cell suspension and analyzed by flow cytometry. Ratios of CD8 ${ }^{+}$and $\mathrm{CD}^{+} \mathrm{FoxP3}^{-}$effector T cells are shown relative to $\mathrm{CD4}^{+} \mathrm{FoxP3}^{+}$Tregs for all mice pooled from 3 independent experiments. Statistical significance between groups for $\mathbf{B}$, $\mathbf{C}$, and $\mathbf{E}$ was determined by ANOVA. ${ }^{*} P<0.05$, ${ }^{* *} P<0.01$, ${ }^{* *} P<0.001$, ${ }^{* * *} P<0.0001$. (F) $P b-C r e 4$, Pten ${ }^{p c-/-S m a d} 4^{p c--}$ mice were treated as in $\mathbf{B}$ beginning at 12 weeks of age and followed for survival. Statistical significance was calculated using the log-rank (Mantel-Cox) test. TIL, tumor-infiltrating lymphocytes.

consistent with the overall reductions in this population noted in Figure 3. Total MDSCs were isolated from these TRAMP-C2 tumors and tested for their capacity to inhibit the proliferation of CFSE-labeled, polyclonally activated $\mathrm{T}$ cells $(\alpha \mathrm{CD} 3 / \alpha \mathrm{CD} 28)$. MDSCs isolated from tumors that had been treated with TH-302 and blockade of CTLA-4 and PD-1 showed reduced ability to inhibit $\mathrm{T}$ cell proliferation relative to Gr-MDSCs from untreated tumors (Figure 4B). Thus, combined hypoxia reduction and checkpoint blockade reduces proliferation and suppressive function of tumor-resident MDSCs. 
To investigate the impact of hypoxia reduction on the functional state of $\mathrm{T}$ cells across the tumor microenvironment as a whole, we established TRAMP-C2 tumors for 2 weeks, treated them with 2 cycles of therapy, and then analyzed their immune infiltrates by flow cytometry. By examining the proliferation marker Ki67, we found that the fraction of $\mathrm{CD}^{+}$effector and $\mathrm{CD}^{+} \mathrm{T}$ cells proliferating in these tumors doubled following combination therapy (Figure 4C). In addition, cytotoxic potential of the CD8 ${ }^{+}$ $\mathrm{T}$ cells in these tumors increased significantly in combinationtreated animals as measured both by elevated per-cell production of granzyme $\mathrm{B}$ and by increases in the percentage of granzyme $\mathrm{B}^{+}$ cells (Figure 4D). Although granzyme B less commonly contributes to actual cytotoxicity in $\mathrm{CD}^{+} \mathrm{T}$ cells, its expression in this compartment was associated with heightened activation and was also elevated with hypoxia reduction and checkpoint blockade (Supplemental Figure 7A).

Recently, a critical role for the rate of $\mathrm{T}$ cell apoptosis in determining success versus failure of antitumor immunity has been demonstrated (23). As $\mathrm{T}$ cells face numerous apoptotic stimuli under hypoxia (13), we sought to determine whether hypoxia reduction and checkpoint blockade improved their survival in this environment. Mice were implanted with TRAMP-C2 tumors in $30 \%$ Matrigel and treated beginning on day 21 for 1 cycle of therapy. The day after conclusion of therapy, mice were injected with pimonidazole (Hypoxyprobe) and euthanized 6 hours later so that hypoxia-exposed versus unexposed populations could be discriminated. Purified CD8 ${ }^{+} \mathrm{T}$ cells from hypoxic areas of prostate tumors showed high-level staining for activated caspase-3 (a hallmark of apoptotic death), while those from mice treated with TH-302 and checkpoint blockade evidenced significantly lower levels of cell death (Figure 4E).

Significant percentages of $\mathrm{CD}^{+} \mathrm{T}$ cells resident in the tumor microenvironment are bystanders lacking in tumor-specificity and capacity to productively contribute to tumor immunity (24). To determine changes in functional phenotype of largely tumor-specific $\mathrm{CD}^{+} \mathrm{T}$ cells, we analyzed those expressing significant levels of the costimulatory molecule 4-1BB (25). TRAMP-C2 tumor-bearing mice were treated with a single cycle of therapy as above, $\mathrm{CD}^{+} \mathrm{T}$ cells were isolated and activated with the Leukocyte Activation Cocktail with Golgi Plug (BD Biosciences) for 5 hours, and then the 4-1BB-expressing subset was analyzed by flow cytometry. Hypoxia reduction and checkpoint blockade resulted in heighted activation in this tumor-specific $\mathrm{CD}^{+} \mathrm{T}$ cell subset based on CD44 expression (Figure 4F). Consistent with the overall $\mathrm{CD}^{+}$population, these $4-1 \mathrm{BB}-$ expressing $\mathrm{CD} 8^{+} \mathrm{T}$ cells also proliferated more robustly (Supplemental Figure 7B). Production of the effector cytokines IFN- $\gamma$ and TNF- $\alpha$ was also significantly enhanced in tumors from TH-302 and $\alpha \mathrm{CTLA}-4 / \alpha \mathrm{PD}-1$-treated mice (Figure 4G). Overall, hypoxia reduction combined with checkpoint blockade qualitatively improved the tumor-specific $\mathrm{CD}^{+} \mathrm{T}$ cell compartment in terms of activation, proliferation, and effector cytokine production.

Hypoxia can suppress $\mathrm{T}$ cell metabolism in numerous ways that impair overall antitumor immunity (26). We isolated $\mathrm{T}$ cells from TRAMP-C2 prostate tumors treated with a single cycle of TH-302 and checkpoint blockade and assayed their mitochondrial function and mass by flow cytometry using MitoTracker Deep
Red FM (a cell-permeable red fluorescent dye whose accumulation depends on mitochondrial membrane potential) and their glycolytic potential by measurement of glucose uptake using fluorescent 2-NBD-glucose (2-NBDG). Both $\mathrm{CD}^{+}$and $\mathrm{CD} 4^{+} \mathrm{T}$ cells showed signs of increased mitochondrial respiration, but with unchanged $\left(\mathrm{CD}^{+}\right)$or slightly reduced $\left(\mathrm{CD} 8^{+}\right)$glycolytic activity (Supplemental Figure 7, C and D). While glycolysis can promote enhanced $\mathrm{T}$ cell cytotoxicity, multiple lines of evidence suggest that improvement of $\mathrm{T}$ cell function and antitumor potential correlates with enhanced mitochondrial respiration and oxidative phosphorylation (27-29).

Having seen that hypoxia reduction and checkpoint blockade reduced the proliferation and suppressive capacity of MDSCs while augmenting the activation and effector function of $\mathrm{T}$ cells, we sought to examine the impact of therapy on the TRAMP-C2 prostate tumor cells themselves. We implanted tumors and analyzed them under the same conditions as above, and found that long after all $\mathrm{TH}-302$ had cleared, tumor cell proliferation was significantly diminished in the remaining hypoxic zones of these cancers (Supplemental Figure 7E). In sum, TH-302 and checkpoint blockade diminished MDSC and tumor proliferation while increasing expansion of highly functional intratumoral $\mathrm{T}$ cell populations.

Reduction of tumor hypoxia impairs the capacity of prostate tumors to replenish their suppressive myeloid stroma. Tumors recruit immature myeloid cells and polarize them to become suppressive MDSCs and TAMs via a variety of factors enriched in hypoxic microenvironments, including VEGF, reactive oxygen species, TGF- $\beta$, IL-6, prostaglandin $\mathrm{E}_{2}, \mathrm{GM}-\mathrm{CSF}$, and G-CSF $(30,31)$. We hypothesized that loss of hypoxic zones might, therefore, compromise the capacity of prostate tumors to recruit and suppressively polarize new myeloid stroma. To test this, we established large TRAMP-C2 tumors (32 days), treated them with a single cycle of therapy, adoptively transferred immature bone marrow myeloid cells from congenically marked mice, and then examined the frequency and phenotype of these cells within the previously treated tumors 60 hours after transfer (Figure 5A). Mice that had been previously treated with hypoxia prodrug and checkpoint blockade were only half as efficient at polarizing the adoptively transferred myeloid progenitors into Gr-MDSCs relative to untreated animals (0.53× Gr-MDSCs of CD45.1 ${ }^{+}$myeloid; $\left.P=0.001\right)$ (Figure $5 \mathrm{~B})$. Further, the cells that bear the markers of Gr-MDSCs

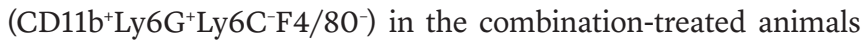
showed incomplete suppressive polarization, as they failed to fully upregulate arginase I $(0.79 \times$ mean fluorescence intensity; $P=0.03)$ and to downregulate MHC II expression (2.03 $\times$ mean fluorescence intensity; $P=0.003$ ) (Figure $5 \mathrm{C}$ ). These data suggest long-term defects in the capacity of these tumors to replenish their Gr-MDSC compartment, which could be related to persistent loss of hypoxia. We hypothesize that the lack of defect in Gr-MDSC polarization in mice receiving $\mathrm{TH}-302$ alone versus the combination in this setting may reflect reestablishment of hypoxia over the week prior to analysis in which these mice received no drug versus maintenance of hypoxia reduction by the expanded proinflammatory immune infiltrate in the presence of checkpoint blockade.

Hypoxia reduction sensitizes spontaneously arising prostate tumors to checkpoint blockade. Prostate tumors in TRAMP mice develop in the dorsolateral prostate and metastasize to the 
periaortic nodes and lungs, with occasional metastases to the kidney, adrenal gland, and bone as early as 12 weeks (15). TRAMP mice develop castration resistance at varied rates, but nearly $100 \%$ are fully androgen-independent by 20 weeks (16). On the B6 background, TRAMP mice develop approximately $80 \%$ adenocarcinomas and 20\% neuroendocrine tumors, but if moved to a B6/FVB $F_{1}$ background they will develop $100 \%$ neuroendocrine cancers (17). We waited until 16 weeks of age to begin treating TRAMP mice (B6 background), at which point they have carcinoma in situ as well as local metastatic disease in most animals. Mice were treated with 3 cycles of therapy and then followed until 36 weeks of age, at which point they were sacrificed and their prostates weighed to assess tumor burden (Figure 6A). When all tumors, both neuroendocrine (triangles) and adenocarcinomas (circles), are considered, as well as all mice regardless of whether they lived to 36 weeks (red indicates death prior to 36 weeks), it is apparent that checkpoint blockade has no impact on tumor progression in this model of prostate cancer (Figure 6B). Because of the rapid progression of neuroendocrine tumors, we were not able to obtain tumor weights for some animals that died before 36 weeks and represented tumors from these animals at the average weight of all neuroendocrine tumors that we were able to measure (5,157 mg). While TH-302 appears to slow the progression of adenocarcinomas in this setting, it has no impact on neuroendocrine disease. The combination of TH-302 and blockade of CTLA- 4 and PD-1, however, has a striking therapeutic effect, with all animals showing tumor control or modest levels of progression at 36 weeks ( $P \leq 0.001$ compared with all groups). This degree of tumor control more than 3 months after the end of therapy is unprecedented in this model. When this study is limited only to mice with adenocarcinomas and only to mice that survive to the 36-week sacrifice, a condition that underrepresents $\mathrm{TH}-302$ tumor sizes, as many more of those animals die before 36 weeks in comparison with combination treatment, it remains clear that the combination of hypoxia-targeted therapy and checkpoint blockade $(877.6 \mathrm{mg})$ controls tumor progression in this model better than checkpoint blockade alone $(2,227 \mathrm{mg} ; \mathrm{P}<0.0001)$ or $\mathrm{TH}-302$ alone $(1,470$ mg; $P=0.0099$ ) (Figure 6C).

While neuroendocrine tumors are known to develop less frequently in the TRAMP model than adenocarcinomas $(20 \%$ neuroendocrine), they develop sooner and progress more rapidly. Between the antibody-treated, TH-302-treated, and untreated TRAMP mice, we observed 23 neuroendocrine tumors versus 58 adenocarcinomas, which represents an insignificant deviation from the predicted frequency based on the published data (Figure $6 \mathrm{D})$. On the other hand, in 23 mice treated with the combination of TH-302 and dual checkpoint blockade, we have yet to observe a single neuroendocrine tumor. This highly significant $(P<0.001)$ underrepresentation of neuroendocrine disease in this group relative to both expected norms and our observed frequency in the other groups suggests that combination therapy can slow and/or block the progression of these tumors, and may even be able to eliminate them altogether.

TRAMP prostate cancer grows diffusely throughout the prostate, making it impossible to grossly distinguish tumor from normal tissue. With this caveat in mind, we analyzed the prostate-infiltrating lymphocytes of TRAMP mice sacrificed at the 36-week time point above from 3 independent experiments. Even nearly 4 months after their last treatment, TRAMP mice that had received TH-302 and CTLA-4/PD-1 blockade showed elevated ratios of both $\mathrm{CD}^{+} \mathrm{T}$ cells to Tregs and $\mathrm{CD}^{+}$effector $\mathrm{T}$ cells to Tregs in their prostates (Figure 6E). While there was a slight trend toward elevated $\mathrm{CD}^{+} / \mathrm{MDSC}$ ratios in the combination-treated animals as well, these differences were no longer significant so far removed from treatment (Supplemental Figure 8A). To test the more immediate impact of therapy in these animals, we treated 30-week-old TRAMP mice with TH-302 and checkpoint blockade for 9 days and then isolated and analyzed their prostate-infiltrating lymphocytes at day 11 ( $n=2,3$ mice per group). In this setting, we observed trends toward higher $\mathrm{CD}^{+}$and $\mathrm{CD} 8^{+} \mathrm{T}$ cell proliferation in the treated mice consistent with our observations in TRAMP-C2 (Supplemental Figure 8B). In addition, we observed a significant drop in monocytic MDSC proliferation and trends toward reduced Gr-MDSC and TAM expansion (Supplemental Figure 8C). While also not statistically significant with the numbers of animals available, $\mathrm{CD}^{+} / \mathrm{MDSC}$ ratios in this setting appeared to improve with treatment (Supplemental Figure 8D). Overall, hypoxia reduction and checkpoint blockade appeared to durably improve effector $\mathrm{T}$ cell/suppressive Treg ratios in TRAMP prostates, and showed signs of MDSC reduction and $\mathrm{T}$ cell expansion that mirrored our observations in the TRAMP-C2 model.

Mice bearing the $\mathrm{Pb}$-Cre $4, \mathrm{Pten}^{\mathrm{pc-}-\mathrm{Smad}} 4^{\mathrm{pc-/}}$ genotype develop aggressive, invasive adenocarcinoma of the prostate by 11 weeks of age, and nearly all die by 32 weeks of age (32). We sought to evaluate the impact of combination checkpoint blockade and hypoxia reduction in these animals that develop tumors with earlier onset and more rapid progression than TRAMP mice due to the distinct molecular drivers in this model. Mice were treated starting at 12 weeks of age for 3 cycles of therapy (last treatment day 115) and then monitored for survival. Combination-treated animals showed significantly improved survival versus untreated animals (Figure 6F). The therapeutic effect in these animals was pronounced during therapy and for some time after; however, over time, these animals progressed with a kinetic approaching that of untreated mice. This progression following discontinuation of therapy is likely due to rapid emergence of new tumors in this model that lack significant antigenic identity with their original disease and, thus, are immune to memory $\mathrm{T}$ cell responses generated during the first round of therapy.

\section{Discussion}

A major impediment to widespread efficacy of $\mathrm{T}$ cell immune checkpoint blockade is the lack of $\mathrm{T}$ cell infiltration observed in "cold" tumors that has been associated with poor prognosis (33). Similarly, hypoxia has been associated with poor prognosis in prostate cancer and many other tumors $(5,10,34)$. We report that hypoxic zones of prostate tumors represent centers of immune and immunotherapy resistance from which $\mathrm{T}$ cells are largely excluded, even in the context of combination checkpoint blockade. Using the hypoxia prodrug TH-302 to reduce tumor hypoxia and potentially also release tumor antigen, we show that hypoxia reduction increases the sensitivity of TRAMP-C2 tumors to checkpoint blockade. We find that TH-302 shows little cooperativity with $\alpha \mathrm{PD}-1$ alone; however, the addition of $\alpha$ PD -1 to $\alpha$ CTLA- 4 augments anti- 
tumor immune responses. We interpret these data to show that depletion of Tregs and enhancement of $\mathrm{T}$ cell priming and expansion are necessary prerequisites to elicit sufficient tumor infiltration by PD-1 $1^{+}$effector T cells to reveal benefit with PD-1 blockade. Unlike TRAMP-C2, which does respond well to checkpoint blockade alone, spontaneous prostate tumors in TRAMP mice are completely unresponsive to CTLA-4/PD-1 blockade, reflecting the lack of response of metastatic prostate cancer patients. In this setting, hypoxia ablation potently sensitizes TRAMP tumors to checkpoint blockade, with mice demonstrating low to absent tumor burden even 4 months after withdrawal of therapy. Especially given the advanced stage of disease at which therapy was initiated in these animals, the therapeutic efficacy of this combination provides a strong rationale for clinical translation.

Suppressive myeloid cells, particularly MDSCs, concentrate in hypoxic zones in these tumors and form a potent barrier to tumor immunity. Reduction of hypoxia with $\mathrm{TH}-302$ not only has an immediate effect on diminishing MDSC frequency and density within these tumors, but also causes persistent defects in the capacity of these tumors to replenish their suppressive myeloid stroma through recruitment and suppressive polarization of new Gr-MDSCs. Further, we find that the capacity of the remaining MDSCs to suppress T cells in mice receiving the TH-302 and checkpoint blockade combination is reduced. Loss of this immune inhibitory barrier reverses the highly suppressive ratio of MDSCs to $\mathrm{CD}^{+} \mathrm{T}$ cells present in untreated tumors, allows these $\mathrm{T}$ cells to infiltrate and survive in formerly hypoxic areas from which they were excluded, and allows the infiltrating effector $\mathrm{T}$ cells to proliferate at twice their prior rate. $\mathrm{CD} 8^{+} \mathrm{T}$ cells in combination-treated tumors also show higher granzyme B expression, higher proliferation and CD44 expression, and greater effector cytokine production. These T cells appear to evade killing by the drug owing to their absence from areas of deep hypoxia, relatively low proliferation absent checkpoint blockade, and capacity to infiltrate hypoxic areas only as they are breaking down and becoming less competent to activate TH-302. Also, both ifosfamide (which shares the active moiety of TH-302) and the related chemotherapeutic cyclophosphamide have demonstrated preferential cytotoxicity for Tregs while sparing effector T cells (35, 36). Further investigation is warranted to determine whether this mechanism translates to other hypoxic tumors that rely on suppressive myeloid stroma to mediate $\mathrm{T}$ cell exclusion and immunotherapy resistance, such as pancreatic cancer and head and neck cancer, particularly the highly hypoxic basal subtype.

An earlier study demonstrated that inhaled hyperoxia was also able to sensitize lung tumors to immune checkpoint blockade (37). We find this study highly supportive of our findings, although its mechanistic focus was on reduction of immune-suppressive extracellular adenosine levels. We plan to investigate the role of adenosine reduction in response to hypoxia reduction in our system in the future, including whether some of the myeloid cell populations that are diminished are contributing to adenosine accumulation. Another highly supportive study was recently published showing a limited capacity of metformin to reduce tumor hypoxia and thereby sensitize a number of murine transplantable tumors to PD-1 blockade (38).

We demonstrate the capacity of targeted hypoxia reduction to sensitize multiple preclinical models of prostate cancer to $\mathrm{T}$ cell checkpoint blockade; however, we have not yet directly addressed the clinical potential of this approach. A recent study of gene signatures that correlate with resistance to PD-1 antibody therapy in melanoma patients found that hypoxia gene sets were enriched in nonresponding patients (39). These observations further support clinical investigation of hypoxia ablation as a mechanism to overcome lack of response to checkpoint blockade. We have therefore opened a phase I clinical trial to explore the capacity of the combination of TH-302 and ipilimumab in this regard at our institution across a number of checkpoint-resistant indications (NCT03098160; ClinicalTrials.gov).

\section{Methods}

Animals. Eight-week-old male C57BL/6, FVB/N, and B6.RAG ${ }^{-/-}$mice were purchased from The Jackson Laboratory. The animals were maintained in a specific pathogen-free environment at the institutional animal facility. All procedures were conducted in accordance with the guidelines established by the University of Texas MD Anderson Cancer Center Institutional Animal Care and Use Committee. TRAMP transgenic mice were also purchased from The Jackson Laboratory, maintained as homozygotes, and then bred to C57BL/6 mice to generate heterozygous progeny used in experiments. $\mathrm{Pb}$-Cre4, Pten $^{p c-/}$ Smad $4^{p c-/}$ mice were provided by the laboratory of Ronald DePinho. At various experimentally indicated time points after tumor challenge and/or immunization, animals were sacrificed according to institutional guidelines.

Cell lines and reagents. The TRAMP-C2 tumor cell line was provided by Norman Greenberg, and all experiments in this study were performed using passage 21-22 tumor cells. TRAMP-C2 cells were maintained as previously described (40). The MyC-CaP cell line was obtained directly from ATCC (ATCC CRL-3255) and used at passage 3 from that stock for all studies.

Therapeutic antibodies and drugs. Therapeutic antibodies were purchased from BioXcell at less than 1 endotoxin unit per milliliter (1 EU/ $\mathrm{ml}$ ) of LPS and included hamster anti-mouse CTLA-4 (9H10 at $100 \mu \mathrm{g} /$ dose) and rat anti-mouse PD-1 (RMP1-14 at $250 \mu \mathrm{g} /$ dose). TH-302 was provided by Threshold Inc., prepared each week in $1 \times$ PBS solution, and used at a dose of $50 \mathrm{mg} / \mathrm{kg}$. Ifosfamide was prepared using the same protocol and dosing $(50 \mathrm{mg} / \mathrm{kg})$.

Prostate tumor treatment experiments. Mice were injected with a single-cell suspension of $1 \times 10^{6} \mathrm{TRAMP}-\mathrm{C} 2$ cells per animal s.c. on the right flank as described previously (41). For MyC-CaP, $2 \times 10^{5}$ cells were injected, and treatment was initiated at day 21 . For tumor infiltrate analysis experiments, TRAMP-C2 cells were implanted in 30\% Matrigel (Corning). Mice received TH-302 at $50 \mathrm{mg} / \mathrm{kg} / \mathrm{d}$ i.p. for 5 straight days beginning 7 days after implantation for TRAMP-C2 treatment studies. CTLA4 and PD-1 blocking antibodies were administered i.p. on days 1, 4, and 7 of each TH-302 cycle. For TRAMP-C2 and MyC-CaP therapeutic and analytic experiments, mice received 2 cycles of therapy with a week of rest in between, while TRAMP and Pb-Cre4, Pten ${ }^{p c-s m a d} 4^{p c-1}$ spontaneous prostate tumor mice received 3 cycles of therapy each with a week of rest in between beginning at 16 weeks of age for TRAMP and 12 weeks of age for $\mathrm{Pb}$-Cre4, $\mathrm{Pten}^{p c-1-S m a d} 4^{p c-/}$.

For TRAMP-C2 and MyC-CaP, tumor growth was measured using a caliper to determine the diameter: longest surface length $(a)$, width $(b)$, and depth $(c)$; and tumor size was expressed as volume $(a \times b \times c)$. Mice were euthanized when the tumor volume reached $1,000 \mathrm{~mm}^{3}$. TRAMP transgenic mice were monitored until 36 weeks of age, at 
which point they were sacrificed and their prostates weighed to assess tumor burden. For mice that died or demonstrated distress or undue tumor burden before 36 weeks of age, every effort was made to recover tumor in a timely fashion for weighing, and all mice were grossly examined to determine whether they carried adenocarcinomas or neuroendocrine carcinomas.

Adoptive transfer of immature myeloid cells. TRAMP-C2 tumors were implanted as described above. Thirty-two days after implantation (tumor size 100-250 $\mathrm{mm}^{3}$ ), animals were treated, or left untreated, for 1 cycle of therapy and then rested for an additional 2 days. $\mathrm{CD} 11 \mathrm{~b}^{+} \mathrm{CD}^{-} \mathrm{CD} 19^{-}$immature myeloid cells were isolated from the bone marrow of B6.SJL mice (The Jackson Laboratory) by FACS using a BD Aria III. CD $45.1^{+}$cells $\left(2 \times 10^{6}\right)$ were then injected i.v. into the CD45.2+ TRAMP-C2 tumor-bearing mice. Sixty hours later, TRAMP-C2 tumors were isolated from these mice and analyzed by flow cytometry with the transferred cells identified as CD $45.1^{+}$.

Tumor-infiltrating lymphocyte isolation. On day 30 after s.c. tumor challenge, mice were sacrificed and tumors were harvested to characterize cell-mediated antitumor responses. Briefly, tumors were digested in X-Vivo-15 (Lonza) supplemented with Collagenase $\mathrm{H}$ (Sigma-Aldrich) and DNase (Roche) and incubated at $37^{\circ} \mathrm{C}$, $5 \% \mathrm{CO}_{2}$ for 30 minutes before being filtered through a $70-\mu \mathrm{m}$ cell strainer. Viable immune cells were enriched through density gradient separation over Histopaque 1119 (Sigma-Aldrich) with a short spin (10 minutes) at $1,000 \mathrm{~g}$ to minimize loss of myeloid populations. Average $\mathrm{CD} 45^{+}$cell percentage after Ficoll separation for untreated TRAMP-C2 tumors $(n=20)$ with this protocol is $8.5 \%$ of viable singlets by flow cytometry. For cell density calculations, samples are counted for viable single cells after disruption but before Ficoll separation on a ViCell counter (Millipore). The percentage of total cells of a given population measured by flow cytometry is then multiplied back against the total cell count to give the total number of those cells. This number is then divided by the mass to derive density (number of cells per milligram of tumor).

Flow cytometry analysis. Samples were fixed using the Foxp3/ Transcription Factor Staining Buffer Set (Affymetrix) and then stained with up to 18 antibodies at a time from BioLegend, BD Biosciences, Affymetrix, Santa Cruz Biotechnology, and Thermo Fisher Scientific. Flow data were collected on a 5-laser, 18-color BD LSR II cytometer and analyzed using FlowJo version 7.6.5 (Tree Star).

Immunofluorescence staining and imaging. Mouse tissues were collected and embedded in Tissue-Tek OCT Compound (Sakura). The embedded tissues were then flash-frozen in liquid nitrogen and sectioned at the MD Anderson Histology Core. The sectioned tissue was fixed with acetone for 10 minutes, permeabilized with the FoxP3 staining kit (eBioscience) for 10 minutes, and blocked with Superblock (Thermo Fisher Scientific) for 15 minutes at room temperature. The samples were stained with antibodies in 2\% BSA, 0.2\% Triton X-100 in PBS at room temperature for 30 minutes and, after being washed in PBS, mounted with ProLong Gold Antifade reagent (Invitrogen). For imaging of hypoxia, pimonidazole (Hypoxyprobe) was administered i.v. to mice 3-6 hours before euthanasia so that hypoxia could be imaged in tumor sections by immunofluorescence staining with antipimonidazole adduct FITC-conjugated antibody (Hypoxyprobe). Fluorescence microscopy was performed using a TCS SP8 laser-scanning confocal microscope equipped with lasers for 405-, 458-, 488-, 514-, 568-, and 642-nm wavelengths (Leica Microsystems Inc.).
For quantitation of $\mathrm{CD}^{+} \mathrm{T}$ cell infiltration into tumors, cells in at least 10 fields per tumor were counted. At least 2 tumors per condition were analyzed. The average $\mathrm{CD}^{+} \mathrm{T}$ cell number per field was calculated.

MDSC suppression assay. Mice were injected s.c. with a single-cell suspension of $1 \times 10^{6}$ TRAMP-C2 cells per animal. Beginning 21 days following implantation, mice were treated with $\mathrm{TH}-302$ in combination with $\alpha$ CTLA- $4+\alpha$ PD- 1 for 2 cycles of therapy. CD $11 b^{+} \mathrm{Gr}-1^{+}$MDSCs were sorted from TRAMP-C2 tumors and cocultured with $\alpha \mathrm{CD} 3$ / $\alpha \mathrm{CD} 28$-activated T cells for 72 hours. T cell proliferation was measured by CFSE dilution. FlowJo 7.6.5 (Tree Star) was used to analyze data.

Metabolic profiling of $T$ cells. Mice were injected s.c. with a singlecell suspension of $1 \times 10^{6}$ TRAMP-C 2 cells per animal. Beginning 21 days after implantation, mice were treated with TH-302 in combination with $\alpha$ CTLA $-4+\alpha$ PD -1 for 1 cycle of therapy. The day after conclusion of therapy, mice were injected with fluorescently labeled 2-NBD-glucose (2-NBDG; Cayman Chemical) i.v. 30 minutes before euthanasia, and glucose uptake was measured by flow cytometry. MitoTracker Deep Red FM staining (Invitrogen) was used to assess total mitochondrial mass.

Characterization of $T$ cell effector function. Mice were injected s.c. with a single-cell suspension of $1 \times 10^{6}$ TRAMP-C 2 cells per animal. Beginning 21 days after implantation, mice were treated with $\mathrm{TH}-302$ in combination with $\alpha$ CTLA- $4+\alpha$ PD- 1 for 1 cycle of therapy. The day after conclusion of therapy, mice were euthanized and viable immune cells enriched through density gradient separation over Histopaque 1119 (Sigma-Aldrich). CD8 ${ }^{+}$ $\mathrm{T}$ cells were isolated from immune cells via negative selection through magnetic beads using a MACS CD8 ${ }^{+} \mathrm{T}$ Cell Isolation Kit (Miltenyi Biotec). $\mathrm{CD}^{+} \mathrm{T}$ cells were activated for 5 hours using Leukocyte Activation Cocktail with Golgi Plug (BD Biosciences) and analyzed using flow cytometry. FlowJo 7.6.5 (Tree Star) was used to analyze data.

Statistics. Computations for all statistical analyses were conducted in GraphPad Prism version 6 for Windows and SPSS. Oneway ANOVAs were applied to test the differences between multiple groups for continuous variables. Overall survival distributions were estimated using the method of Kaplan and Meier and compared using the Mantel-Cox (log-rank) test.

For tumor growth curves, the linear mixed model was used to analyze the longitudinal tumor size data with consideration of withinmouse correlations. This model evaluated the time trends of tumor sizes across different treatment groups by including interaction terms between the treatment and time. The proportion test was applied to compare the predicted and observed frequencies of adenocarcinoma and neuroendocrine tumor development in the TRAMP model. Graphs show mean \pm SEM unless otherwise indicated. $P$ values less than 0.05 were considered statistically significant.

Study approval. All animal studies were approved by the MD Anderson Cancer Center Institutional Animal Care and Use Committee (Houston, Texas, USA) under protocol 00001378-RNO0/1.

Data availability. All data generated or analyzed during this study are included in this published article (and its supplementary information files). Any reasonable requests for more detailed descriptions of data included herein will be honored by the corresponding author.

\section{Author contributions}

PJ, MA, AL, and MAC designed research. PJ, MA, AL, TB, PB, JS, $\mathrm{CA}, \mathrm{CN}, \mathrm{ARJ}, \mathrm{YS}, \mathrm{KS}, \mathrm{SB}$, and MAC performed research. PJ, MA, $\mathrm{AL}, \mathrm{AZ}, \mathrm{TZ}$, and MAC performed confocal imaging and associated 
analysis. PJ, MA, AL, TB, CA, CN, and MAC analyzed data. GW provided transgenic animals and untreated survival data. NL, JN, PJ, MA, and MAC performed statistical analyses and power calculations. PJ, MA, AL, and MAC wrote the manuscript.

\section{Acknowledgments}

MAC was supported in this work by a Cancer Research Institute CLIP Grant and by the MD Anderson Prostate Cancer SPORE (P50 CA140388) Career Development Program. These studies are currently being supported by an Individual Investigator Research Award from the Cancer Prevention and Research
Institute of Texas (CPRIT RP170399). The MD Anderson Immunology Imaging Core and Flow Cytometry Core facilities assisted in these studies and are supported by the S10 RR029552 (NIH) and MD Anderson instrumentation grants. Threshold Inc. kindly provided TH-302 for this work as well as consultation on its use.

Address correspondence to: Michael A. Curran, University of Texas MD Anderson Cancer Center, Department of Immunology, 1515 Holcombe Boulevard, Unit 901, Houston, Texas 77054, USA. Phone: 713.563.3286; Email: mcurran@mdanderson.org.
1. Curran MA, Montalvo W, Yagita H, Allison JP. PD-1 and CTLA-4 combination blockade expands infiltrating $\mathrm{T}$ cells and reduces regulatory $\mathrm{T}$ and myeloid cells within $\mathrm{B} 16$ melanoma tumors. Proc Natl Acad Sci US A. 2010;107(9):4275-4280.

2. Wolchok JD, et al. Nivolumab plus ipilimumab in advanced melanoma. $N$ Engl J Med. 2013;369(2):122-133.

3. Kwon ED, et al. Ipilimumab versus placebo after radiotherapy in patients with metastatic castration-resistant prostate cancer that had progressed after docetaxel chemotherapy (CA184043): a multicentre, randomised, double-blind, phase 3 trial. Lancet Oncol. 2014;15(7):700-712.

4. Royal RE, et al. Phase 2 trial of single agent Ipilimumab (anti-CTLA-4) for locally advanced or metastatic pancreatic adenocarcinoma. J Immunother. 2010;33(8):828-833.

5. Saggar JK, Yu M, Tan Q, Tannock IF. The tumor microenvironment and strategies to improve drug distribution. Front Oncol. 2013;3:154.

6. Corzo CA, et al. HIF-1 $\alpha$ regulates function and differentiation of myeloid-derived suppressor cells in the tumor microenvironment. J Exp Med. 2010;207(11):2439-2453.

7. Dang EV, et al. Control of T(H)17/T(reg) balance by hypoxia-inducible factor 1 . Cell. 2011;146(5):772-784.

8. Ben-Shoshan J, Maysel-Auslender S, Mor A, Keren $\mathrm{G}$, George J. Hypoxia controls $\mathrm{CD} 4{ }^{+} \mathrm{CD} 25^{+}$regulatory T-cell homeostasis via hypoxia-inducible factor-1 $\alpha$. Eur J Immunol. 2008;38(9):2412-2418.

9. Becker JC, Andersen MH, Schrama D, Thor Straten P. Immune-suppressive properties of the tumor microenvironment. Cancer Immunol Immunother. 2013;62(7):1137-1148.

10. Ragnum HB, et al. The tumour hypoxia marker pimonidazole reflects a transcriptional programme associated with aggressive prostate cancer. Br J Cancer. 2015;112(2):382-390.

11. Vaupel P, Höckel M, Mayer A. Detection and characterization of tumor hypoxia using $\mathrm{pO} 2$ histography. Antioxid Redox Signal. 2007;9(8):1221-1235.

12. Motz GT, et al. Tumor endothelium FasL establishes a selective immune barrier promoting tolerance in tumors. Nat Med. 2014;20(6):607-615.

13. Chouaib S, Noman MZ, Kosmatopoulos K, Curran MA. Hypoxic stress: obstacles and opportunities for innovative immunotherapy of cancer. Oncogene. 2017;36(4):439-445.

14. Duan JX, et al. Potent and highly selective hypoxia-activated achiral phosphoramidate mustards as anticancer drugs. JMed Chem. 2008;51(8):2412-2420.

15. Gingrich JR, et al. Metastatic prostate cancer in a transgenic mouse. Cancer Res. 1996;56(18):4096-4102.

16. Tang Y, Wang L, Goloubeva O, Khan MA, Zhang B, Hussain A. Divergent effects of castration on prostate cancer in TRAMP mice: possible implications for therapy. Clin Cancer Res. 2008;14(10):2936-2943.

17. Chiaverotti T, et al. Dissociation of epithelial and neuroendocrine carcinoma lineages in the transgenic adenocarcinoma of mouse prostate model of prostate cancer. Am J Pathol. 2008;172(1):236-246.

18. Ellwood-Yen K, et al. Myc-driven murine prostate cancer shares molecular features with human prostate tumors. Cancer Cell. 2003;4(3):223-238.

19. Watson PA, Ellwood-Yen K, King JC, Wongvipat J, Lebeau MM, Sawyers CL. Context-dependent hormone-refractory progression revealed through characterization of a novel murine prostate cancer cell line. Cancer Res. 2005;65(24):11565-11571.

20. Quezada SA, Peggs KS, Curran MA, Allison JP. CTLA4 blockade and GM-CSF combination immunotherapy alters the intratumor balance of effector and regulatory T cells. JClin Invest. 2006;116(7):1935-1945.

21. Curran MA, Kim M, Montalvo W, Al-Shamkhani A, Allison JP. Combination CTLA-4 blockade and 4-1BB activation enhances tumor rejection by increasing T-cell infiltration, proliferation, and cytokine production. PLoS One. 2011;6(4):e19499.

22. Casazza A, et al. Impeding macrophage entry into hypoxic tumor areas by Sema3A/Nrp1 signaling blockade inhibits angiogenesis and restores antitumor immunity. Cancer Cell. 2013;24(6):695-709.

23. Horton BL, Williams JB, Cabanov A, Spranger S, Gajewski TF. Intratumoral CD $8^{+} \mathrm{T}$-cell apoptosis is a major component of t-cell dysfunction and impedes antitumor immunity. Cancer Immunol Res. 2018;6(1):14-24.

24. Simoni $\mathrm{Y}$, et al. Bystander $\mathrm{CD}^{+} \mathrm{T}$ cells are abundant and phenotypically distinct in human tumour infiltrates. Nature. 2018;557(7706):575-579.

25. Ye Q, et al. CD137 accurately identifies and enriches for naturally occurring tumor-reactive $\mathrm{T}$ cells in tumor. Clin Cancer Res. 2014;20(1):44-55.

26. Vuillefroy de Silly R, Dietrich PY, Walker PR. Hypoxia and antitumor $\mathrm{CD}^{+} \mathrm{T}$ cells: an incompatible alliance? Oncoimmunology. 2016;5(12):e1232236

27. Geiger R, et al. L-Arginine modulates $\mathrm{T}$ cell metabolism and enhances survival and anti-tumor activity. Cell. 2016;167(3):829-842.e13.

28. Sukumar M, et al. Inhibiting glycolytic metabolism enhances $\mathrm{CD}^{+} \mathrm{T}$ cell memory and antitumor function. J Clin Invest. 2013;123(10):4479-4488.

29. Scharping NE, et al. The tumor microenvironment represses $\mathrm{T}$ cell mitochondrial biogenesis to drive intratumoral $\mathrm{T}$ cell metabolic insufficiency and dysfunction. Immunity. 2016;45(3):701-703.

30. Gabrusiewicz K, et al. Glioblastoma-infiltrated innate immune cells resemble MO macrophage phenotype. JCI Insight. 2016;1(2):e85841.

31. Marvel D, Gabrilovich DI. Myeloid-derived suppressor cells in the tumor microenvironment: expect the unexpected. JClin Invest. 2015;125(9):3356-3364.

32. Ding Z, et al. SMAD4-dependent barrier constrains prostate cancer growth and metastatic progression. Nature. 2011;470(7333):269-273.

33. Fridman WH, Pagès F, Sautès-Fridman C, Galon J. The immune contexture in human tumours: impact on clinical outcome. Nat Rev Cancer. 2012;12(4):298-306.

34. Wilson WR, Hay MP. Targeting hypoxia in cancer therapy. Nat Rev Cancer. 2011;11(6):393-410.

35. Madondo MT, Quinn M, Plebanski M. Low dose cyclophosphamide: mechanisms of T cell modulation. Cancer Treat Rev. 2016;42:3-9.

36. Brown JS, Sundar R, Lopez J. Combining DNA damaging therapeutics with immunotherapy: more haste, less speed. Br J Cancer. 2018;118(3):312-324.

37. Hatfield SM, et al. Immunological mechanisms of the antitumor effects of supplemental oxygenation. Sci Transl Med. 2015;7(277):277ra30.

38. Scharping NE, Menk AV, Whetstone RD, Zeng X, Delgoffe GM. Efficacy of PD-1 blockade is potentiated by metformin-induced reduction of tumor hypoxia. Cancer Immunol Res. 2017;5(1):9-16.

39. Hugo W, et al. Genomic and transcriptomic features of response to anti-PD-1 therapy in metastatic melanoma. Cell. 2016;165(1):35-44.

40. Foster BA, Gingrich JR, Kwon ED, Madias C, Greenberg NM. Characterization of prostatic epithelial cell lines derived from transgenic adenocarcinoma of the mouse prostate (TRAMP) model. Cancer Res. 1997;57(16):3325-3330.

41. Curran MA, Allison JP. Tumor vaccines expressing flt3 ligand synergize with ctla- 4 blockade to reject preimplanted tumors. Cancer Res. 2009;69(19):7747-7755. 